\title{
A Rotating Azimuthally Distributed Auroral Current System on Saturn Revealed by the Cassini Spacecraft
}

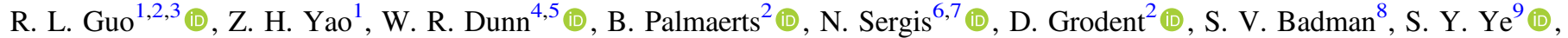

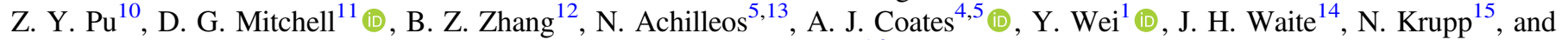 \\ M. K. Dougherty ${ }^{16}$ (iD \\ ${ }^{1}$ Key Laboratory of Earth and Planetary Physics, Institute of Geology and Geophysics, Chinese Academy of Sciences, Beijing 100029, People's Republic of China \\ Ruilong.guo@uliege.be, z.yao@ucl.ac.uk \\ ${ }^{2}$ Laboratory for Planetary and Atmospheric Physics, STAR Institute, Université de Liège, Liège B-4000, Belgium \\ ${ }^{3}$ Laboratory of Optical Astronomy and Solar-Terrestrial Environment, Institute of Space Sciences, School of Space Science and Physics, Shandong University, \\ Weihai, Shandong, People's Republic of China \\ ${ }^{4}$ Mullard Space Science Laboratory, University College London, Holmbury St. Mary, Dorking RH5 6NT, UK \\ ${ }^{5}$ Centre for Planetary Sciences at UCL/Birkbeck, London WC1E 6BT, UK \\ ${ }^{6}$ Office of Space Research and Technology, Academy of Athens, Athens 106 79, Greece \\ ${ }^{7}$ Institute of Astronomy, Astrophysics, Space Applications and Remote Sensing, National Observatory of Athens, Athens 118 10, Greece \\ ${ }^{8}$ Department of Physics, Lancaster University, Lancaster LA1 4YB, UK \\ ${ }^{9}$ Department of Earth and Space Sciences, Southern University of Science and Technology, Shenzhen 518055, People's Republic of China \\ ${ }^{10}$ School of Earth and Space Sciences, Peking University, Beijing 100871, People's Republic of China \\ ${ }^{11}$ Applied Physics Laboratory, Johns Hopkins University, Laurel, MD 20723, USA \\ ${ }^{12}$ Department of Earth Sciences, University of Hong Kong, Hong Kong, People's Republic of China \\ ${ }^{13}$ Department of Physics and Astronomy, University College London, London WC1E 6BT, UK \\ ${ }^{14}$ Southwest Research Institute, San Antonio, TX 78238, USA \\ 15 Max-Planck-Institut für Sonnensystemforschung, Göttingen D-37077, Germany \\ ${ }^{16}$ Faculty of Natural Sciences, Department of Physics, Imperial College London, London SW7 2AZ, UK \\ Received 2021 July 29; revised 2021 September 11; accepted 2021 September 14; published 2021 October 1
}

\begin{abstract}
Stunning aurorae are mainly produced when accelerated electrons travel along magnetic field lines to collide with the atmosphere. The motion of electrons often corresponds to the evolution of a magnetic field-aligned current system. In the terrestrial magnetosphere, the current system is formed at the night-side sector, and thus produces an auroral bulge at night. Due to the different energy sources between Saturn and the Earth, it is expected that their auroral current systems are fundamentally different, although the specific auroral driver at Saturn is poorly understood. Using simultaneous measurements of the aurora, particles, magnetic fields, and energetic neutral atoms, we reveal that a chain of paired currents, each of which includes a downward and an upward current branch, is formed in Saturn's magnetosphere, which generates separated auroral patches. These findings inform similar auroral current structures between the Earth and Saturn, while the difference is that Saturn's unique mass and energy sources lead to a rotational characteristic.
\end{abstract}

Unified Astronomy Thesaurus concepts: Planetary magnetospheres (997); Saturn (1426); Aurorae (2192)

\section{Introduction}

The global magnetic field of a planet can fend off the solarwind particles to form a magnetosphere. Various dynamic processes in the magnetosphere can accelerate particles originating from the solar wind or the natural moons (i.e., volcanos and water vapor). The Earth's magnetosphere is mainly driven by solar-wind activities. Saturn's magnetosphere is usually considered to be a rotationally driven system, but it could be substantially modulated by solar-wind activities. The rotationally driven processes stretch the magnetosphere to form a disk-like magnetic configuration, i.e., the magnetodisk. Beyond the magnetodisk, the solar wind shapes the magnetosphere to form a stretched magnetotail on the night side. A number of observations at Earth have shown that many fundamental plasma processes like magnetic reconnection and dipolarization serve as the critical mechanisms in producing the field-aligned current (FAC) wedge in the night-side magnetotail and powering the stunning auroral bulges in the ionosphere (Boström 1964; McPherron et al. 1973; Liu et al. 2015). Similarly, in the traditional model for Saturn's and Jupiter's magnetospheres, rotationally driven magnetic reconnection only occurs on the night side. The reconnection site is triggered in the pre-night sector and can extend to the dawn-side magnetopause (Vasyliunas 1983).

Several mysteries regarding Saturn's magnetospheric dynamics can occur at all local times. For example, quasiperiodic energetic electron pulsations are one of the important phenomena in Saturn's magnetosphere and are found at all local times (Mitchell et al. 2016; Palmaerts et al. 2016b; Bader et al. 2019b). The quasi-periodic pulsations correlate with auroral pulsations and auroral hiss, and the latter is an indication of FACs (Mitchell et al. 2016; Bader et al. 2019b). However, the precise explanation of this pulsation has not yet been found. A recent study used a realistic plasma/field model showing that the field line resonance's third and fourth harmonic modes correspond to quasi-periodic $1 \mathrm{hr}$ pulsations (Rusaitis et al. 2021). In addition, a rotating auroral spiral structure was suggested to be related to the recurrence of magnetic dipolarization (Palmaerts et al. 2020). Further, the existence of auroral patches or auroral beads at Saturn may imply localized dynamic processes like shear flow ballooning instability (Radioti et al. 2019). These studies suggest that the magnetospheric dynamics driving aurorae in the ionosphere are complex and cannot be explained simply with traditional 
theories, urging an improved model for the global Saturnian auroral processes.

Recent studies of Saturn's magnetosphere revealed that magnetic reconnection can also take place in the day-side magnetodisk (Guo et al. 2018a), which was unexpected given the traditional model. Compared to the traditional night-side reconnection, the length scales of the day-side magnetodisk reconnection are relatively small (Delamere et al. 2015; Guo et al. 2018b). A further study demonstrates that the multiple small-scale reconnection regions are discretely distributed at all local times and are rotating with Saturn's magnetosphere (Guo et al. 2019). Besides the reconnection sites, recurrent magnetospheric dipolarization was also uncovered in Saturn's day-side magnetosphere (Yao et al. 2018). The discoveries of day-side magnetodisk reconnection and dipolarization provide crucial implications for interpreting the day-side auroral emissions on giant planets, which were often attributed to processes taking place on the magnetopause (Radioti et al. 2013). The rotating feature of the reconnection sites (Guo et al. 2019) and the recurrent dipolarization (Yao et al. 2018) also indicate a new cycling model that can advance the understanding of rotating phenomena in Saturn's magnetosphere and ionosphere.

In this Letter, using observational data from Cassini, phenomena in both Saturn's magnetosphere and ionosphere are physically connected. We show that FACs chain the accelerated particles near the magnetosphere equator and auroral emission. More importantly, those chains rotate and act on a larger area than expected according to traditional knowledge, i.e., also on the day side.

\section{Observational Results}

\subsection{Rotating Main Auroral Patches (RMAPs) and Their Magnetospheric Context}

Energetic particles are one of the major products in a magnetospheric region disturbed by various instabilities where particles are energized and the magnetic morphology is distorted. Collisions between the energetic particles and background cold atoms can produce energetic neutral atoms (ENA) that could directly be imaged by the ion and neutral camera (INCA) on the Magnetosphere Imaging Instrument (MIMI) onboard Cassini (Krimigis et al. 2004). Figure 1(a) displays an image of the ENA in Saturn's active magnetosphere on 2008 July 15. The resolution of the ENA image recorded by Cassini was insufficient to reveal the details of the active regions. The field of view (FOV) was also limited and could not cover ENA information from all local times. Fortunately, during this time, the Cassini Ultraviolet Imaging Spectrograph (UVIS) instrument (Esposito et al. 2004) provided contemporaneous auroral counterparts for these magnetospheric events. Figures 1(b)-(d) display three selected auroral images in the southern ionosphere. (The whole consecutive sequence of the auroral images is shown in Figure B1 in the Appendix B. The images are projected on a polar map and viewed from above the north pole. From this point of view, a west-to-east rotation corresponds to a counterclockwise motion).

The successive auroral images in the Appendix B show that a chain of auroral patches distributes in azimuth and rotates with Saturn; these are called rotating main auroral patches (RMAPs) in this study. The RMAPs cover at least half of the main aurora region. The pink dots in the images mark the magnetic footprint of Cassini obtained by using the University
College London/Achilleos-Guio-Arridge magnetodisk model (Achilleos et al. 2010). The ionospheric latitude of the magnetic footprint of Cassini moves slowly from 73.7 to $72^{\circ} .9$ during the $\sim 6 \mathrm{hr}$ of recording by UVIS. In addition to the mapping model, we used an Archimedean spiral equation to account for the bend-back effect of the magnetic field lines, as this substantially improves the local-time (LT) mapping of Cassini's position along the magnetic field lines into the ionosphere. The bend-back effect results in a deviation of $\Delta \mathrm{LT}=24\left(B_{\varphi} / B_{r}\right) \ln \left(\rho_{\text {Cassini }} / \rho_{\text {Enceladus }}\right) / 2 \pi$ ranging from 0.3 to $0.8 \mathrm{hr}$ between Cassini and its magnetic footprint, where $\rho_{\text {Cassini }}$ and $\rho_{\text {Enceladus }}$ are the perpendicular distances of Cassini and Enceladus from the spin axis of Saturn, respectively.

Four distinct auroral patches at the dawn-side polar region are labeled with numbers on the images. Previous literature has reported the spiral structure of auroral patch "1" (Radioti et al. 2015). But the driving mechanism of the global auroral morphology, corresponding to the major energy dissipation processes in Saturn's magnetosphere, is unknown. The averaged angular velocity can be calculated by tracing each auroral patch from image to image. We obtain a value of $\sim 75 \%$ of rigid rotation ( 1 rotation per $\sim 10.7 \mathrm{hr}$ ), similar to the value previously reported (Radioti et al. 2015). The ENA images in the equatorial plane also show corresponding rotating features, as seen in the full sequential records of the ENA images in Figure B2 in the Appendix B. (The ENA images are viewed from the actual location of Cassini, that is, from the southern hemisphere. From this point of view, a west-to-east rotation appears to move in the clockwise direction.) Although the detailed ENA structures cannot be clearly identified due to the limited temporal and spatial resolution of the INCA instrument, both the ENA and the aurora are consistently covering similar local times (see Figure 1(e) and the yellow dashed outlines in Figures 1(a) and (b), bearing in mind the limitation of the FOVs for both INCA and UVIS instruments). Cassini was nearly motionless compared to the rotation of the magnetosphere. When seen in the frame rotating with the magnetosphere, Cassini swept over each of auroral patches (Cassini's trajectory relative to the auroral patches is shown in Figure 1(e)).

The Cassini magnetometer (MAG) instrument (Dougherty et al. 2004) detected a series of magnetic variations (Figure 1(f)). The two curves in Figure 1(f) are the $s_{\perp 1}$ and $s_{\perp 2}$ magnetic components in a mean-field-aligned (MFA) coordinate system (detailed in the figure caption), in which the $s_{\perp 1}$ and $s_{\perp 2}$ directions are perpendicular to the mean magnetic field (averaged over 20 minutes; Yao et al. 2017). The two perpendicular magnetic components suggest parallel currents flowing along the magnetic field lines, i.e., FACs. Figure 1(g) shows the enhancements of the power spectrum density of the electric field detected by the RPWS instrument (Gurnett et al. 2004). The enhanced waves represent auroral hiss and match the large magnetic perturbations, revealing a close relationship between the auroral hiss and FACs, as suggested in a previous study (Gurnett et al. 1983). In addition, the differential flux of $\sim 100 \mathrm{keV}$ electrons (red curve in Figure $1(\mathrm{~g})$, from the $\mathrm{C} 4$ channel of the MIMI-LEMMS) recurrently enhances when recording the auroral hiss. Using simultaneous ENA measurements, UV auroral images, and auroral hiss signals, we can directly distinguish between spatial and temporal variations for these interconnected phenomena in this event, thereby putting forward a new global physical picture to explain all these observations. Here we provide direct evidence that the 

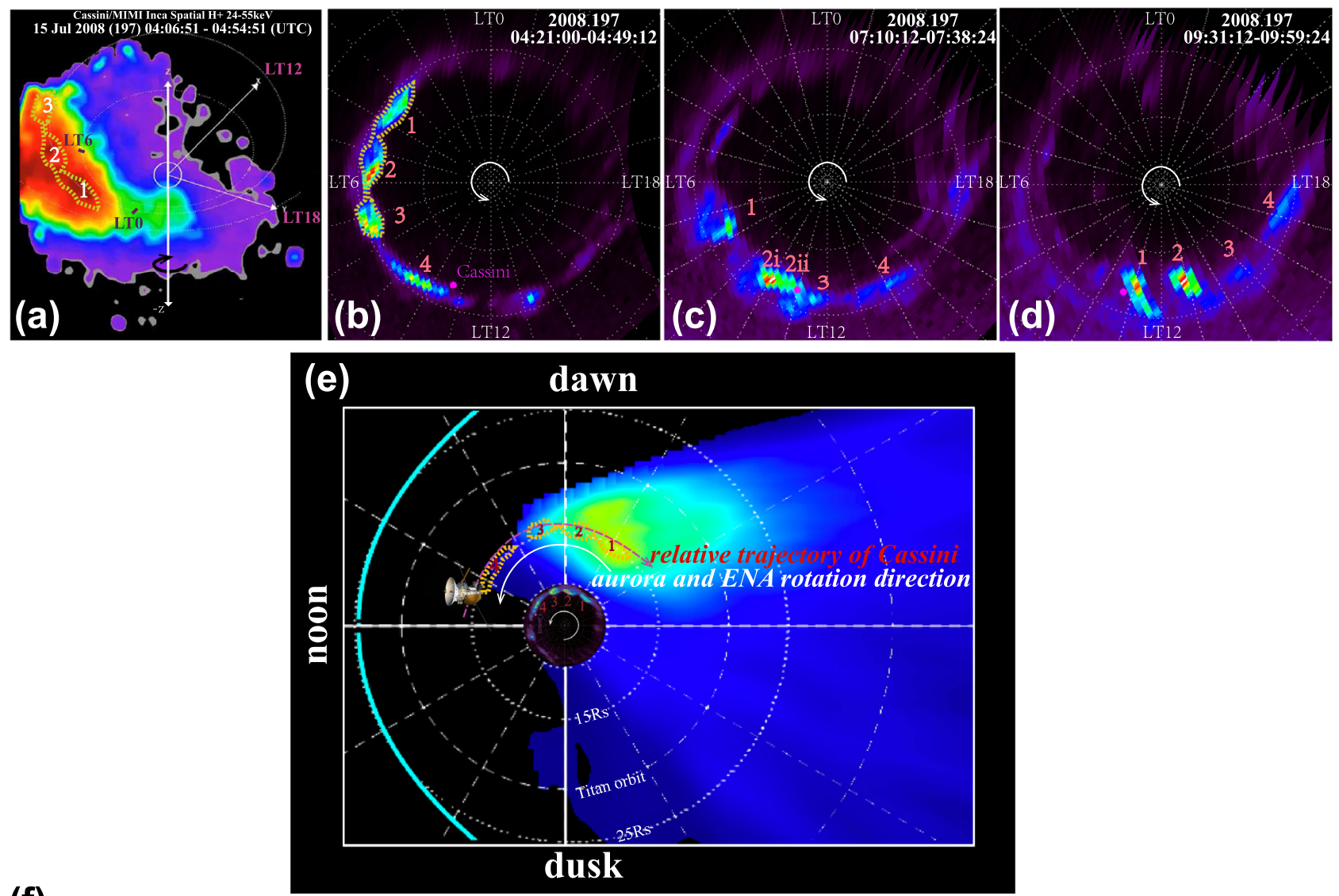

(f)
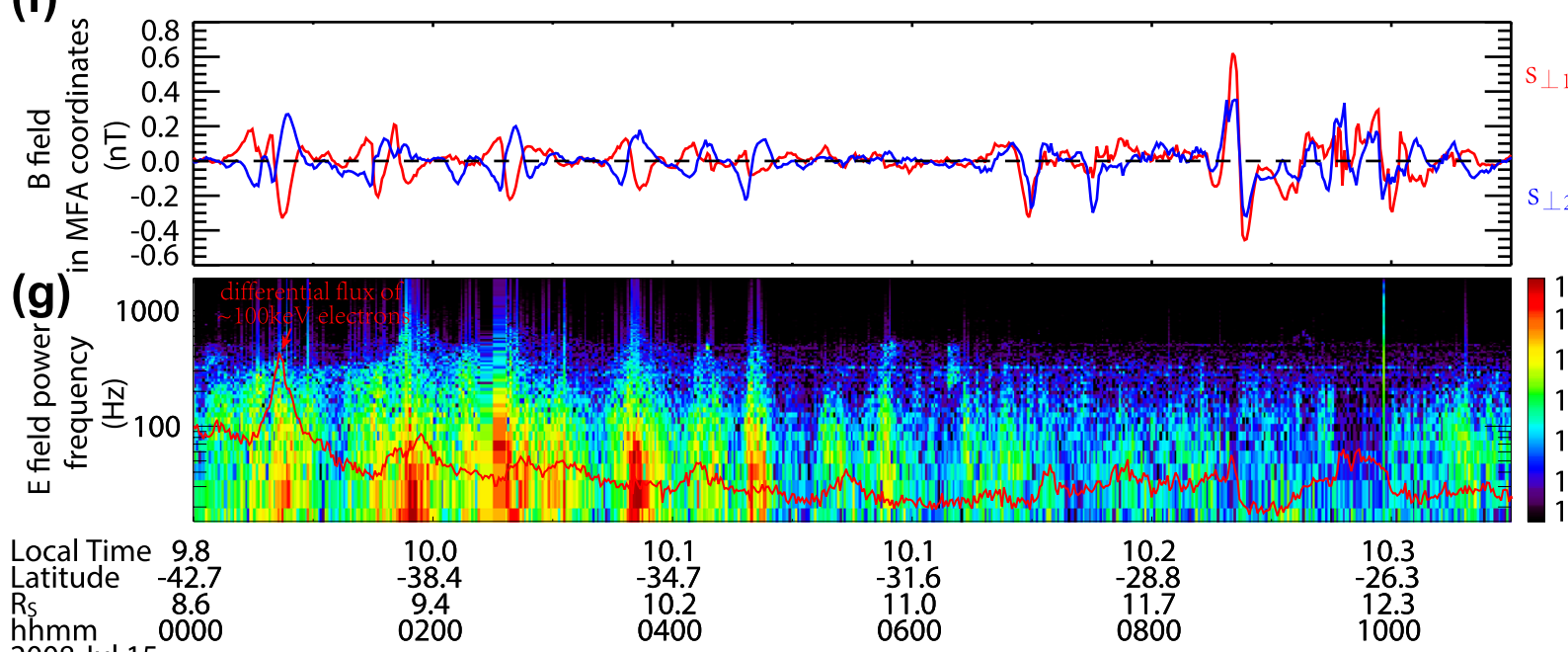

Rs

2008 Jul 0000

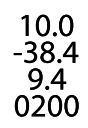

0.0
8.4
.04

200

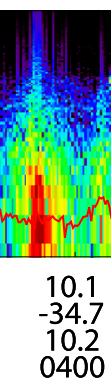

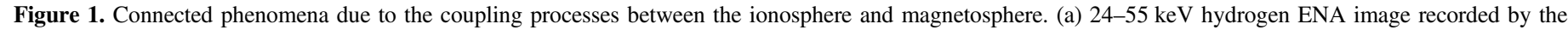

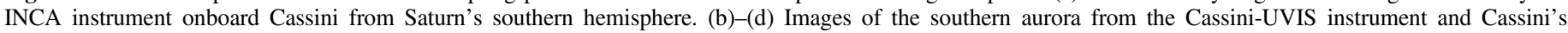

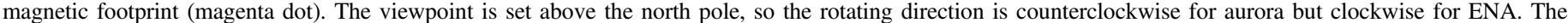

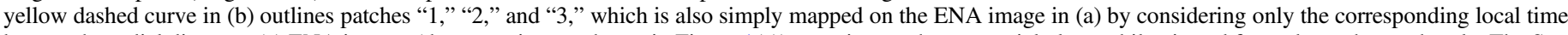

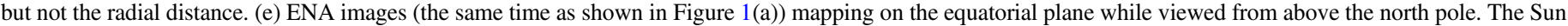

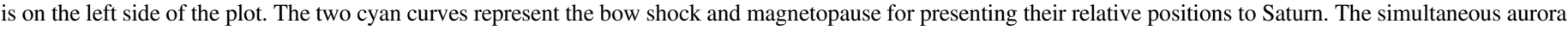

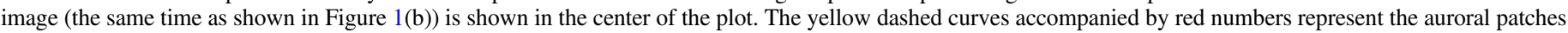

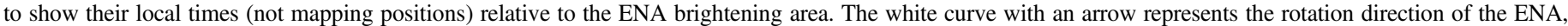

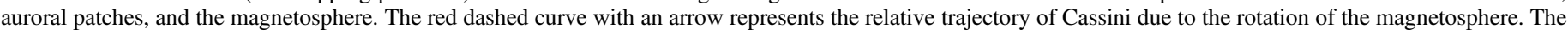

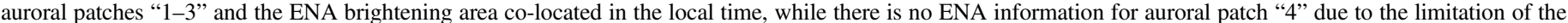

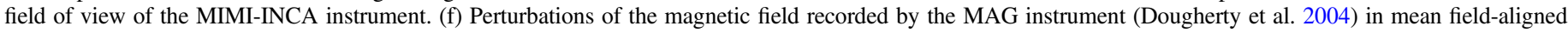

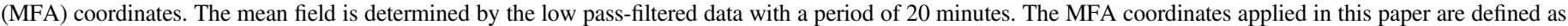

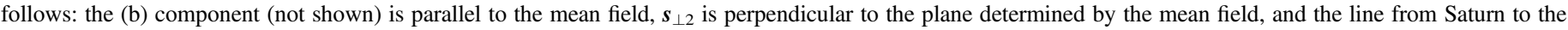

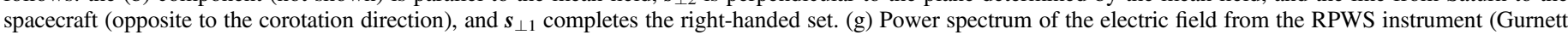

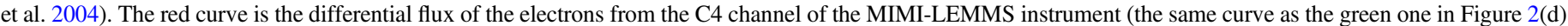
but scaled with a factor of 20 for co-plotting with the electric power spectrogram). 


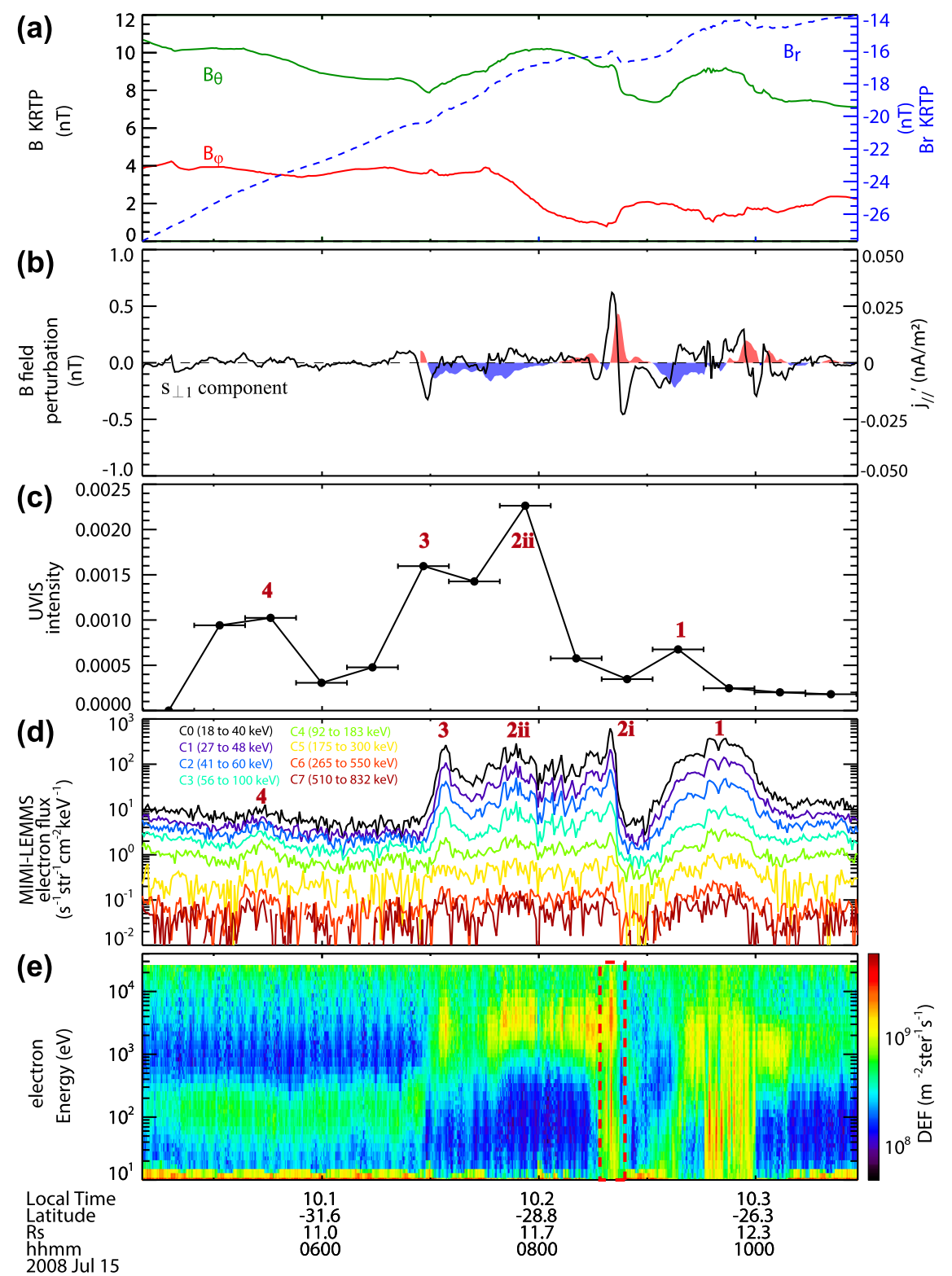

Figure 2. Relationship between FACs, auroral patches, and energetic electron enhancements. (a) $B_{\theta}$ (green), $B_{\varphi}$ (red), and $B_{r}$ (dashed blue) components of the magnetic field in the Kronographic Radial-Theta-Phi coordinates (KRTP, a spherical polar coordinate system). (b) The $\boldsymbol{s}_{\perp 1}$ component of the magnetic field (black curve). The area highlighted in blue/red shows the estimated parallel current density $j_{/}^{\prime}$ with blue indicating negative values (out of the ionosphere) and red indicating positive values (going into the ionosphere). (c) Variation of the auroral intensity measured by the UVIS instrument at the magnetic footprint of Cassini during the rotation of the aurorae (integrated over an area of $2^{\circ} \times 0.4 \mathrm{hr}$ (Latitude $\times \mathrm{LT}$ ) that centered at Cassini's footprint). The horizontal segments show the integration time to obtain each aurora image. (d) Energetic electrons flux from the MIMI-LEMMS instrument. (e) Hot electron spectrum from the CAPS instrument (Young et al. 2004).

energetic electron recurrences associated with RMAPs observed for this interval are due to rotating spatial structures rather than temporal variations.

\subsection{Multiple FAC System Associated with RMAPs}

Cassini flew through regions with evident magnetic field perturbations between 07:00 and 10:00 UT (Figure 2(a)), which correspond to the FACs as indicated by the perpendicular magnetic perturbations in the MFA coordinates. The direction of FACs can be derived by applying the differential form of Ampere's law to the observed magnetic field in Figure 2(a) (detailed in the method section). The estimation is meaningful only when Cassini is passing through the regions of FAC. During the interval plotted in Figure 2, Cassini was not in the magnetodisk, following the criteria defined by Arridge et al. (2008) and described in the Appendix A (see Figure B3(c)). The latitude value of Cassini was lower than $-26^{\circ}$, suggesting that Cassini was also far away from the cusp region. The region where Cassini stayed during the studied interval is the midlatitude magnetosphere. Before the large magnetic perturbation, Cassini's MIMI-LEMMS instrument observed electron fluxes in Figure 2(e) that peaked at an energy of $\sim 100 \mathrm{eV}$ before $\sim 07: 00$ UT. When flying through the magnetic field perturbations, the energy of the peaking flux shows a sudden increase around 07:00 UT, and the fluxes of energetic electrons (Figure 2(d)) are also notably enhanced. The large perturbations of both $B_{\theta}$ and $B_{\varphi}$ magnetic components in Figure 2(a) suggest Cassini flying through a disturbed magnetospheric region. The $B_{r}$ magnetic 
component gradually varied from $\sim-60 \mathrm{nT}$ to $\sim-14 \mathrm{nT}$, suggesting that the sudden electron flux enhancement was not caused by a rapid flapping of the current sheet. All the above pieces of evidence collectively indicate that, when recording the sudden change of hot electron flux, Cassini entered the FACs at mid-latitudes rather than other magnetospheric regions. The estimated FAC density $j_{/ /}^{\prime}$ (see method) is represented by the area highlighted in blue/red in Figure 2(b), with blue indicating negative values (going out of the ionosphere) and red indicating positive values (going into the ionosphere).

The auroral intensity measured by UVIS at Cassini's magnetic footprint in the ionosphere is shown in Figure 2(c). The numbers for the intensity peaks indicate their corresponding auroral patches. The peaks of the auroral intensity generally coincide with the negative currents (electrons flowing into the ionosphere) (Figure 2(b)) and the enhancements of the energetic electron flux (Figure 2(d)). It should be noted that, due to the instrument geometry and limited FOV, the LEMMS instrument was unable to directly detect the electrons traveling along the magnetic field lines during this auroral event. Therefore, some enhancements of energetic electrons (e.g., the populations with velocities nearly perpendicular to the magnetic field lines) may not correspond to auroral emission. Nevertheless, the highly energetic (especially 100s keV) electrons indicate strong acceleration processes in the magnetosphere. The first auroral intensity peak corresponds to auroral patch "4." Unfortunately, unlike patches " $1-3$," Cassini's footprint did not go through the center of the magnetospheric counterpart of auroral patch "4" (see Figures B1 (a)-(c)), so no recognizable heating is seen in the hot electron spectrum (Figure 2(e)). A flux peak of the energetic electrons (Figure 2(d)) (their large gyro-radii allow them to disperse into a large area at their source region) coincides with aurora patch "4" but was relatively weak compared to the signatures detected for patches " $1-3$."

From 06:13 UT to 08:06 UT (see Figures B1(e)-(h) in the Appendix B), the auroral morphology dynamically evolved: auroral patch "2" extended to momentarily merge with patch " 3 " and then separated again. The transient evolution of the active magnetospheric region related to aurora patch "2" resulted in two enhancements of the energetic electron flux labeled "2ii" and "2i." However, Cassini penetrated the FAC flowing into the ionosphere (positive parallel current in Figure 2(b)) when recording electron enhancement " $2 \mathrm{i}$ " (Figure 2(d)) and matched a low UVIS intensity (Figure 2(c)). The electrons with low energies around the $100 \mathrm{eV}$ enhanced at "2i" in Figure 2(e) show antiparallel pitch angles (see Figure B3 (d) in the Appendix B), i.e., coming from the ionosphere, in agreement with the positive FAC current density. In summary, the energetic electron enhancements of " $2 \mathrm{ii}$ " and " $2 \mathrm{i}$ " are related to the FAC system of auroral patch " 2 ." As a consequence of the transient expansion of auroral patch " 2 ," the energetic electron flux during the interval from " 3 " to " $2 \mathrm{i}$ " did not show a significant drop like the one shown in the interval between " $2 \mathrm{i}$ " to " 1 ."

The positive and negative currents appear alternately, suggesting a chain of up-down current pairs associated with the RMAPs. This type of electrical current morphology often exists in the terrestrial auroral region and is characteristic of current wedgelets (Rostoker 1991; Forsyth et al. 2014; Liu et al. 2015), which are small-scale structures compared to the major auroral intensifications, e.g., substorm auroral bulges.
Table 1

List of Patchy or Bead-like Aurora Events

\begin{tabular}{lll}
\hline \hline IONOSPHERE & $\begin{array}{l}\text { MID-LATITUDE } \\
\text { MAGNETOSPHERE } \\
\text { Corresponding Signatures in the Dura- } \\
\text { tion including One Period before and } \\
\text { RMAPs Events }\end{array}$ & $\begin{array}{l}\text { EQter RMAPs } \\
\text { Rotang ENA } \\
\text { Patch }\end{array}$ \\
\hline $2008-197$ & SH, NDEE & yes \\
$2016-229$ & SH, NDEE & yes \\
$2016-231$ & SH & yes \\
$2016-275$ & SH, NDEE & $\ldots$ \\
$2017-232$ & SH, NDEE & yes \\
\hline
\end{tabular}

Note. SH: separated hiss as shown in Figure 1(g); NDEE: non-dispersion energetic electrons; $\cdots$ : no data.

The energetic electrons do not show a discernible energy-time dispersion feature for each peak, indicating that the electrons were not transported from a remote region. The energetic electron flux was only enhanced when encountering the FACs that linked the isolated aurora beads, implying that the acceleration regions were separated in the magnetosphere.

Additionally, Table 1 gives a list of cases showing RMAPs and their contemporaneous recurrent energetic electrons and/or aurora hiss, demonstrating that the interconnections among ENA, multiple FACs, and main auroral patches are systematic in the Saturn system.

\subsection{Recurrence of the Multiple FAC System}

Figures 3(a) and (b) show the comparison of the features in magnetic component $B_{\varphi}$ perturbations and energetic electrons during the UVIS recording intervals (black curves) and the interval $11 \mathrm{hr}$ later (red curves). These plots show a recurrence feature of the structures with a timescale of Saturn's rotation period, implying that the multiple FAC system can last longer than one planetary rotation period. The recurrence of the observational features may also be attributed to the rocking of the magnetosphere and the auroral oval (Bader et al. 2019a), which let Cassini penetrate the FACs during the intervals when the auroral oval was offset toward Cassini's footprint (Arridge et al. 2016). The difference between black and red curves may be attributed to the motion of Cassini (from $\sim 12 R s$ to $\sim 15 R s$ in distance to Saturn's center and from $\sim-30^{\circ}$ to $\sim-15^{\circ}$ in latitude) and/or the temporal evolution of the structures. For example, the transient evolution of auroral patch " 2 " produces a short-lived energetic electron flux peak before 08 UT (black curve in Figure 3(b)), which disappears $11 \mathrm{hr}$ later (red curve in Figure 3(b)). Alongside the similarities between magnetic fields sampled from two planetary rotations (i.e., separated by about one planetary rotation), the energetic ions revealed by the ENA (Figure B2 in Appendix B) also recurred. This provides evidence that the active region may have lasted for more than one rotation period of Saturn and rotated at least once around Saturn to sweep past the spacecraft twice.

\section{Discussion and Summary}

In this study, we report a rotating multiple FAC system in Saturn's magnetosphere. Although it shares a similar morphology with the terrestrial wedgelet current system (Forsyth et al. 2014), we notice that Saturn's multiple FAC systems are substantially different in both spatial and temporal scales. 

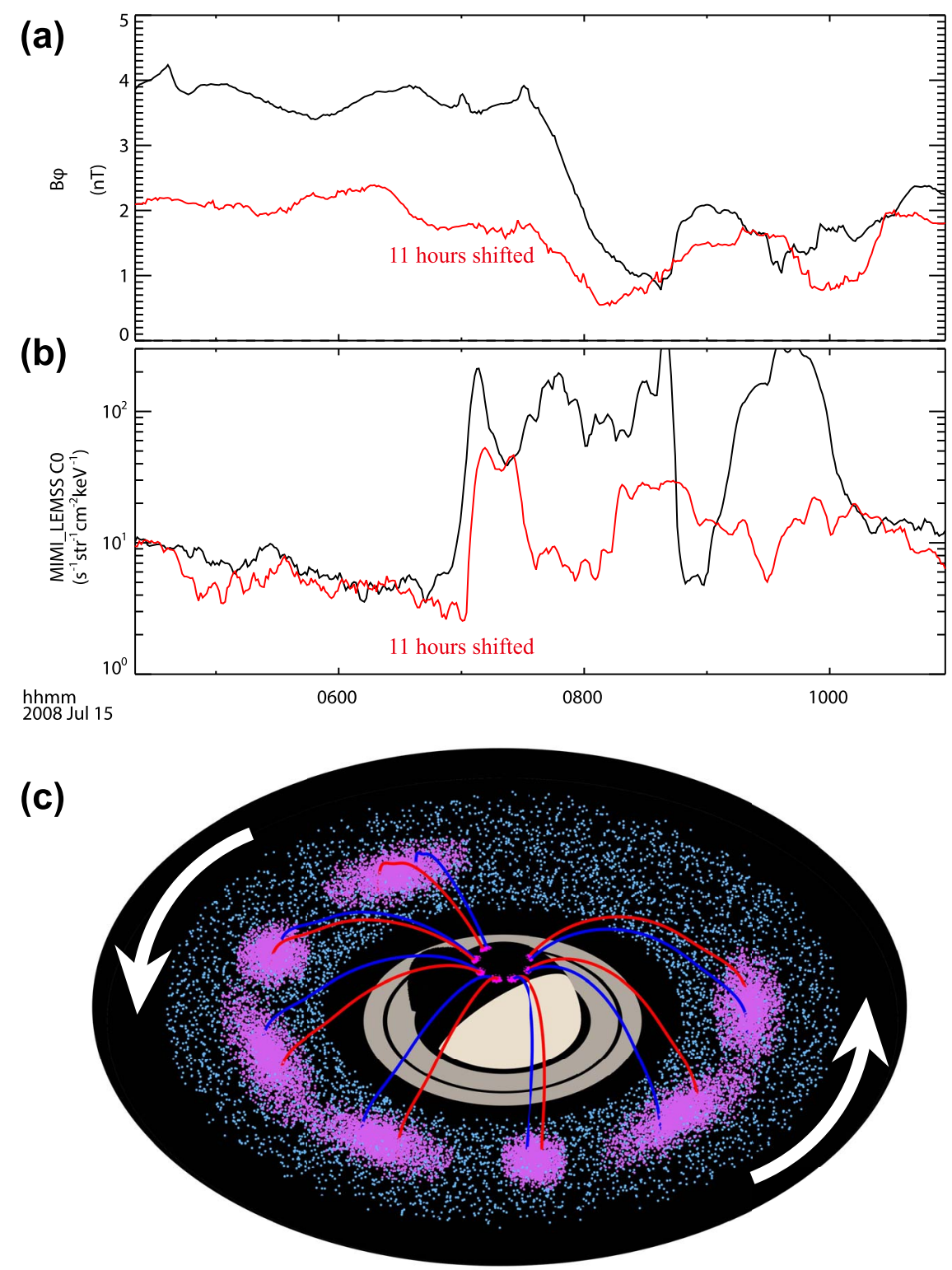

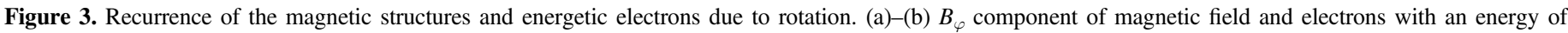

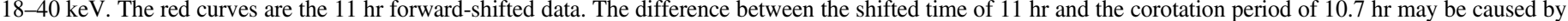

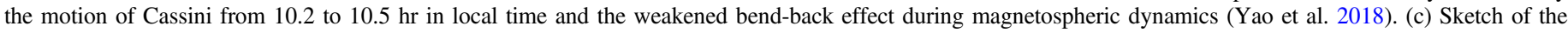

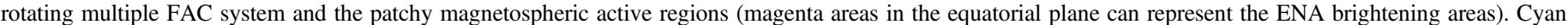

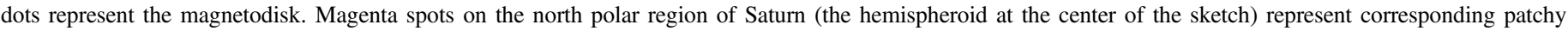

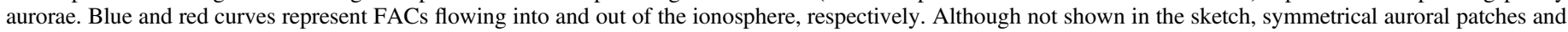
FACs can also be seen in Saturn's south pole.

Figure 3(c) illustrates the connection among the multiple FAC system, the ENA brightening areas, and the main auroral patches. Multiple active regions (magenta areas in the equatorial plane) link multiple auroral patches (magenta spots on the north polar region of Saturn) through complex FAC networks (blue and red curves). The multiple FAC system may cover a larger area than Cassini detected, considering the limited FOV of both UVIS and INCA instruments. The auroral images and the recurrence of auroral hiss suggest that multiple FACs and active regions could be distributed discretely across much of the magnetosphere. Auroral hiss and the corresponding energetic electrons in Figure 1(g) show that Cassini passed through several FACs before recording the UVIS images. The first aurora image in Figure 1(b) also confirms that there are auroral patches duskward of Cassini's footprint, which probably have rotated from the dawn side a few hours ago.

The discovery of the multiple FAC system reported in this study provides a crucial constraint for theoretical investigations of the formation of Saturn's main auroral emission, which is probably also applicable to Jupiter. The recorded recurrence of energetic electrons is often associated with quasi-periodic electron pulsations. They were previously interpreted as temporal effects of the magnetospheric dynamics (Mitchell et al. 2009; Palmaerts et al. 2016a, 2016b; Bader et al. 2019b) or the consequences of dynamical processes on the magnetopause like Kelvin-Helmholtz waves (Masters et al. 2010) or magnetodisk reconnection occurring at dusk (Bader et al. 2019b). During RMAP events, the slowly moving spacecraft 
would cross the relatively fast-rotating flux tubes connected to each auroral patch. Therefore, the rotation effect of the multiple active regions can lead to pulsating features in energetic electron data, e.g., the red curve in Figure 1(g). The planetaryrotation-induced "electron pulsations" would complement the previous physical interpretation on the commonly observed quasi-periodical phenomena.

During the rotation, the aurora is brighter on the dawn side than in the noon sector. The local-time asymmetry in the auroral intensification may be explained by the following. (1) the formation of wedgelet currents is also accompanied by other processes that can contribute to auroral emissions, e.g., Alfvén waves. On the dawn side of the magnetosphere, the shear flow between the corotating magnetosphere and the tailward magnetosheath flow is maximum. Then, the Alfvénic Poynting flux associated with the wedgelets on the dawn side may be greater than at the other local times. (2) The wedgelets may experience a local-time modulation (which is a common feature in giant planets' magnetosphere, e.g., Carbary et al. 2017; Palmaerts et al. 2017) during the rotation, causing asymmetric auroral intensification.

Although the wedgelet current system studied at Earth also contains multiple up-down FAC pairs and generates a chain of bead-like aurorae, it is limited to the night side and cannot extend to the day side. Its lifetime is on the order of a few minutes (Liang et al. 2008; Rae et al. 2009). The large spatial scale and long temporal scale of the multiple FACs on Saturn suggest that the mechanism for explaining the wedgelet current at Earth cannot be applied directly to Saturn's magnetosphere. The recent discoveries of day-side magnetodisk reconnection (Guo et al. 2018a) and dayside magnetic dipolarization (Yao et al. 2018) indicate that the day-side magnetic field lines could be more stretched than previously expected. When the field lines are stretched at all local times, fundamental plasma instabilities, like cross field current instability, sausage instability, and ballooning/centrifugal instability (Lui 2004), may thus develop at any local time. Therefore, multiple FAC systems could be formed on a global scale. Multiple FAC systems could also be a consequence of multiple longstanding small-scale reconnection sites distributed discretely in the magnetodisk (Guo et al. 2019). The multiple active regions existing at all local times can speed up the mass-loss rate in Saturn's magnetosphere. It may be more critical at Jupiter, where internal processes predominantly drive magnetospheric dynamics.

Cassini operations are supported by NASA (managed by the Jet Propulsion Laboratory) and ESA. R.L.G. thanks Japheth Yates and Aikaterini Radioti for their contribution to the discussions on the electron energizations and aurora emission. R.L.G. is supported by the Incoming Post-Docs in Sciences, Technology, Engineering, Materials, and Agrobiotechnology (IPD-STEMA) project from Université de Liège. D.G. and B.P. acknowledge financial support from the Belgian Federal Science Policy Office (BELSPO) via the PRODEX Programme of ESA: 4000130563. Z.Y. was supported by the National Science Foundation of China (grant 42074211) and Key Research Program of the Institute of Geology \& Geophysics CAS (grant IGGCAS-201904). S.V.B. was supported by UK STFC grants ST/V000748/1 and ST/M005534/1. A.J.C. and W.R.D. acknowledge support from UCL-MSSL solar system consolidated grant ST/S000240/1 from STFC, UK. The data from Cassini's MAG, CAPS, MIMI, RPWS, and UVIS instruments onboard the NASA/ESA Cassini spacecraft are available at https://pds-ppi.igpp.ucla.edu/.

\section{Appendix A \\ Estimation of the Current Density for the FAC}

Since the angular velocity of the rotating magnetosphere is much larger than the speed of Cassini relative to Saturn, we may assume that Cassini remained fixed for the duration of an auroral event. Thus the motion of Cassini's footpoint relative to the planetary magnetic field was almost entirely due to planetary rotation. In the spherical polar coordinates, as a preliminary estimation, we assume that $r$ and $\theta$ of Cassini's position were constant while only $\varphi$ was changing. We thus get $\delta \varphi=2 \pi C \delta t / P_{\text {rigid }}$, where $P_{\text {rigid }} \cong 10.7 \mathrm{hr}$ is the rigid rotation period of Saturn and $C \approx 0.75$ is the ratio between the rotation speed of the aurora beads and the rigid rotation speed. The three components of the differential form of Ampere's law are:

$$
\begin{gathered}
\mu_{0} j_{r}=\partial B_{\varphi} / r \partial \theta-\partial B_{\theta} / r \sin \theta \partial \varphi \\
\mu_{0} j_{\theta}=\partial B_{r} / r \sin \theta \partial \varphi-\partial B_{\varphi} / \partial r \\
\mu_{0} j_{\varphi}=\partial B_{\theta} / \partial r-\partial B_{r} / r \partial \theta
\end{gathered}
$$

In the case studied in this paper, we can calculate the terms with $\partial \varphi: \quad j_{r}^{\prime}=-\partial B_{\theta} /\left(\mu_{0} r \sin \theta \partial \varphi\right)$ and $j_{\theta}^{\prime}=\partial B_{r} /$ $\left(\mu_{0} r \sin \theta \partial \varphi\right)$. The combination of these two terms is the total variations in the $\varphi$ direction caused by the current flow, i.e., independent of other directions. We can assume for simplicity that the FAC has a nearly circular transverse section and the variation of the current density inside the FAC during the concerned interval is not too big. For a columnar current structure, different directions of the current flow would cause opposite variation patterns along the $\varphi$ direction. In other words, the combination of $j_{r}^{\prime}$ and $j_{\theta}^{\prime}$ can indicate the direction of the current flow.

Cassini was located in the southern magnetosphere and at the anti-planetward position (Sunward in this case) from the center of the FAC, as indicated by Cassini's magnetic footprint in the auroral images. At this position in the FAC, increasing $r$ and $\theta$ means moving in the direction from the FAC's center to the edge. According to the integral form of Ampere's law, the magnetic field generated by the FAC is stronger at the edge than that near the center. Hence, the increasing $r$ and $\theta$ corresponds to an increase of $\left|B_{\varphi}\right|$. For the FAC parallel to the magnetic field, i.e., $j_{/ /}>0$, the generated $B_{\varphi, \text { FAC }}<0$, and $f=\partial B_{\varphi} /\left(\mu_{0} r \partial \theta\right)$ and $g=\partial B_{\varphi} /\left(\mu_{0} \partial r\right)$ are negative. For the FAC antiparallel to the magnetic field, i.e., $j_{/ /}<0$, the generated $B_{\varphi, \mathrm{FAC}}>0$, and both $f$ and $g$ are positive.

The direction of the averaged magnetic field line is $\boldsymbol{b}_{\text {averaged }}=[-0.66,0.35,0.09]$ in the Kronographic RadialTheta-Phi coordinates when Cassini was inside FACs and did not change too much. Under the assumption that the current was parallel to the mean magnetic field line, $j_{\varphi}$ is much smaller than the other two components and can be neglected in the estimation. The parallel current density $j_{/ /}=\boldsymbol{j} \bullet \boldsymbol{b}_{\text {averaged }}=$ $-0.66 j_{r}+0.35 j_{\theta}=-0.66 j^{\prime}{ }_{r}+0.35 j^{\prime}{ }_{\theta}-(0.66 f+0.35 g)$. Combining the above analysis, the term of $-(0.66 f+0.35 g)$ has the same sign as $j_{/ /}$in this case, i.e., the lack of $f$ and $g$ does not affect the sign of $j_{/ /}$. In Figure 2(b), we plot the $j_{/}^{\prime}=b_{r} j_{r}^{\prime}+b_{\theta} j_{\theta}{ }^{\prime}$ by the colored areas to show the directions of the FACs. Since several assumptions are applied during the analysis, the current density amplitude is imprecise and the error is hard to estimate. Even though when the magnitude is large, the sign of $j_{/ /}^{\prime}$ could be reliable to represent the sign of 
$j_{/ /}$, the sign is what we are really concerned with in this study. The antiparallel low-energy electrons in Figures 2(e) and B3 (d) are consistent with the positive FAC. Additionally, the sign of $j_{/ 1}^{\prime}$ is consistent with the bipolar signature of the $s_{\perp 1}$ magnetic component when crossing FAC transversely: the bipolar feature changing from positive to negative (from negative to positive) corresponds to positive (negative) $j_{/ /}$.

The estimation is valid under a quasi-steady state. The transient expansion of aurora patch " 2 " from 06:13 UT to 08:06 UT would bring relatively large uncertainty to the calculation and may also disturb the bipolar signature of the $s_{\perp 1}$ magnetic component. Despite these, the existence of multiple FACs is explicit according to the measured magnetic perturbations.
The $s_{\perp 1}$ and $s_{\perp 2}$ components in the MFA coordinates only represent part of the FAC-induced magnetic variations. The 20 minute averaged magnetic field is not exactly the background field but contains part of the FAC-induced magnetic field. The total FAC-induced magnetic variation is on the order of several nTs, as shown in Figure 2(a). Despite this, the $s_{\perp 1}$ and $s_{\perp 2}$ components are helpful to demonstrate the finite structures of the FAC system.

\section{Appendix B}

Figures B1 and B2 show the whole consecutive sequence of the auroral images and ENA images, respectively. Figures B3 shows the criteria for classifying current-sheet-like magnetic field and the electron pitch angle information.

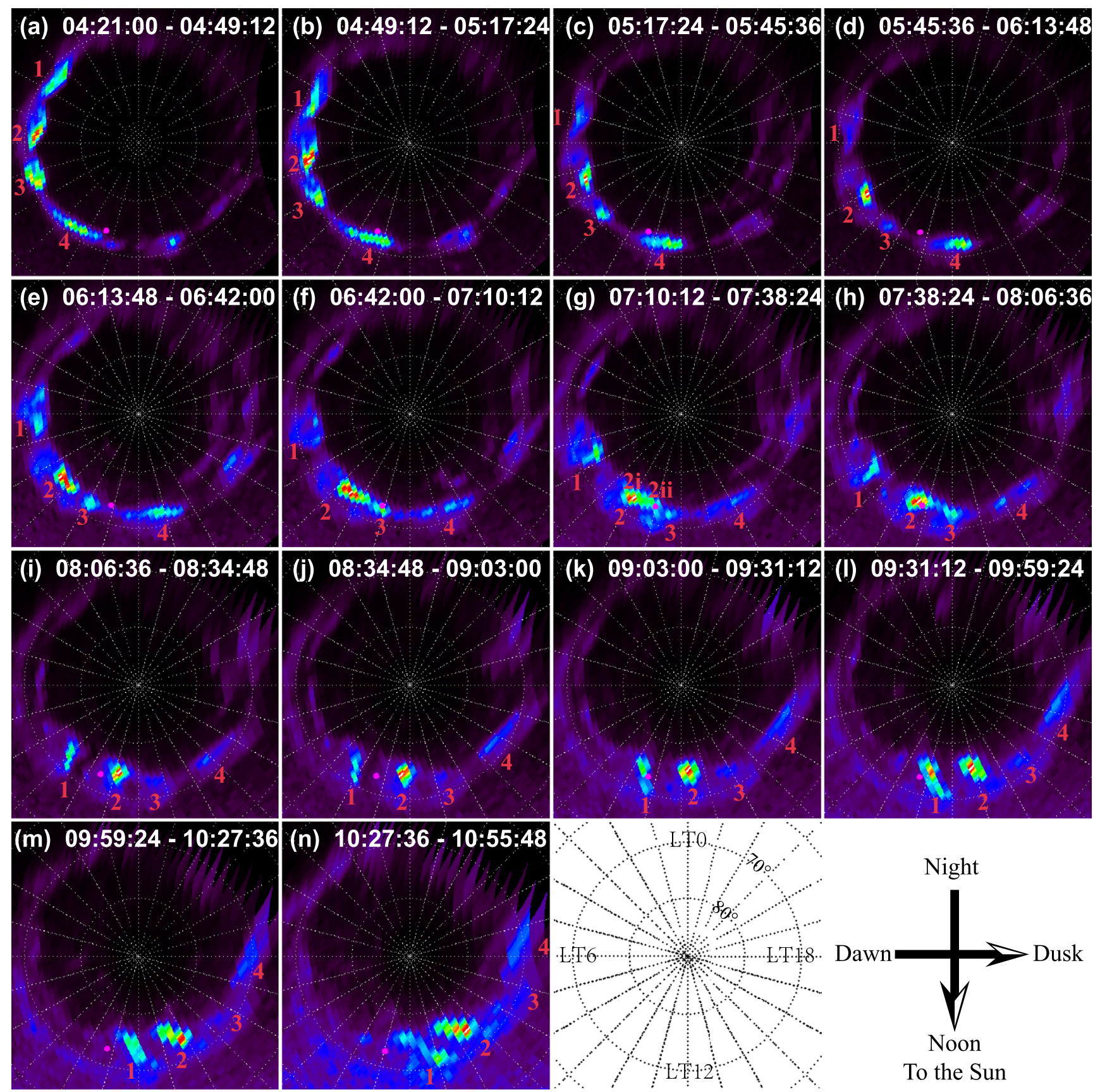

Figure B1. Aurora images observed by the UVIS instrument onboard Cassini. The aurorae were on the southern polar ionosphere, while the images were viewed above the north pole. The magenta dot in each image represents Cassini's magnetic footprint. A transient explosion of patch "2" starts at (e) and finishes at (h), which lasts about 1 hr. 


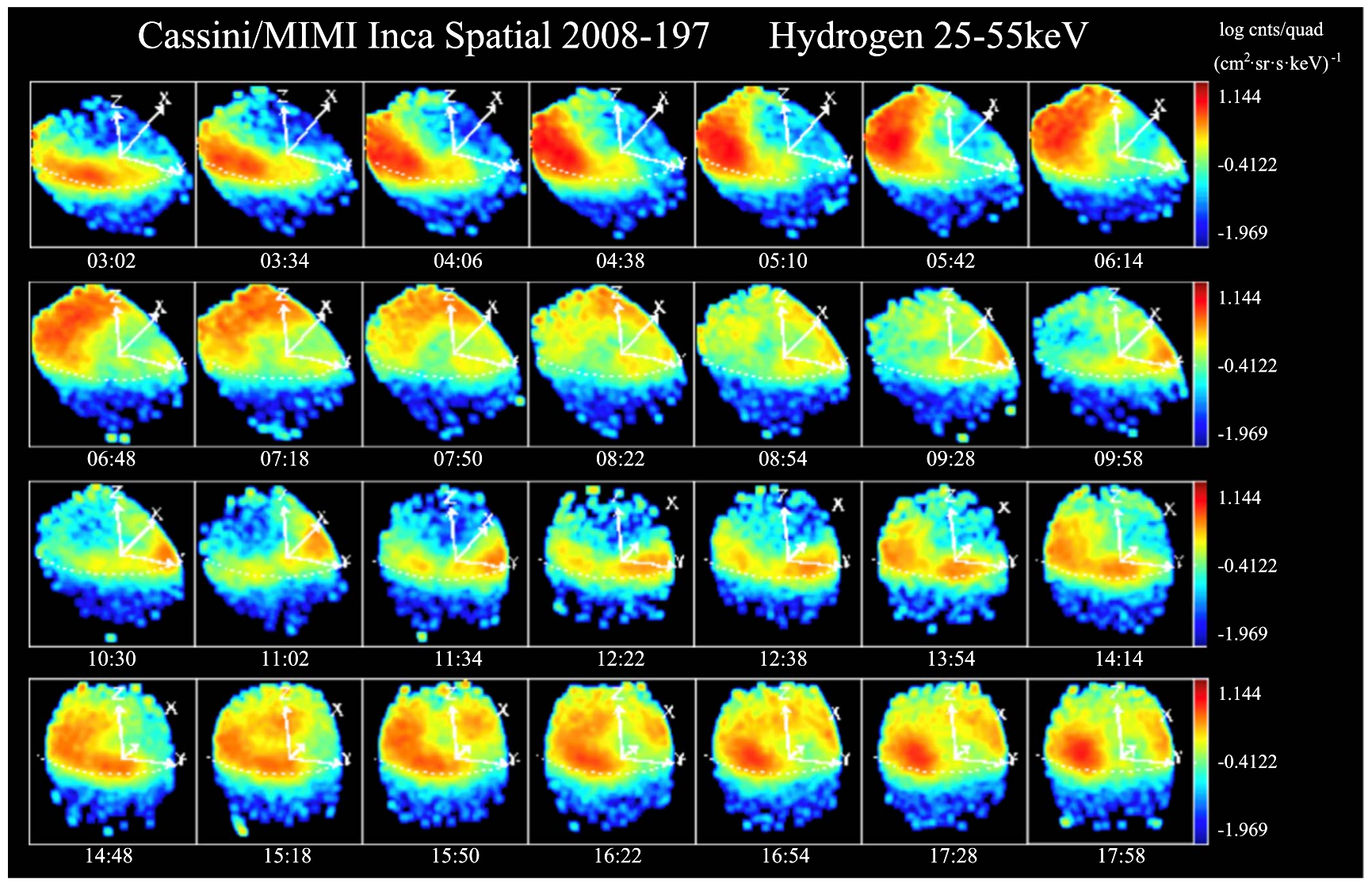

Figure B2. ENA images on 2008 July 15. Cassini was in the southern hemisphere when these images were taken. The time sequence shows the ENA rotated back after one rotation period. 

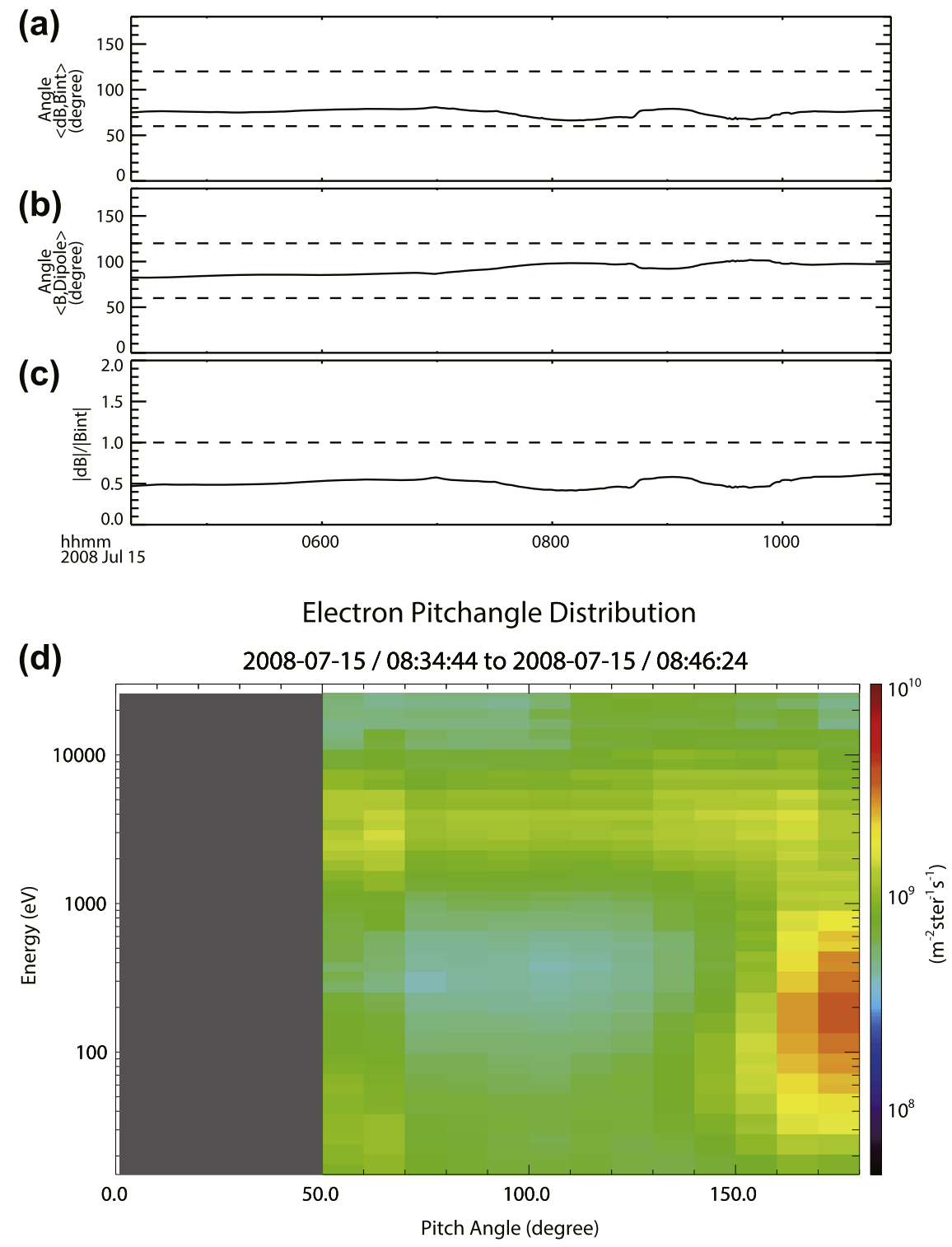

Figure B3. (a)-(c) Criteria for classifying current-sheet-like magnetic field (Arridge et al. 2008). The two angles in (a) and (b) lie within 60 to 120 degrees, but in (c), the ratio between the magnetic deviation and the internal field is less than one. The deviation $|\boldsymbol{d} \boldsymbol{B}|=\left|\boldsymbol{B}_{\text {observation }}-\boldsymbol{B}_{\text {internal }}\right|$, where $\boldsymbol{B}_{\text {observation }}$ is the observed magnetic field and $\boldsymbol{B}_{\text {internal }}$ is the internal magnetic field obtained from the Cassini 11 model (Dougherty et al. 2018). (d) The electron pitch-angle energy distribution generated during the interval is highlighted by the two red dashed vertical lines. The distributions indicate that the electrons with energies of $\sim 100 \mathrm{eV}$ are antiparallel, i.e., coming from the ionosphere. Note that we do not know the parallel fluxes due to instrumental limitations.

\section{ORCID iDs}

R. L. Guo @ ittps://orcid.org/0000-0002-7125-0942

W. R. Dunn (1) https://orcid.org/0000-0002-0383-6917

B. Palmaerts (i) https://orcid.org/0000-0003-2762-4334

N. Sergis 1 h https://orcid.org/0000-0003-4144-8699

D. Grodent (ii) https://orcid.org/0000-0002-9938-4707

S. Y. Ye iㅏ https://orcid.org/0000-0002-3064-1082

D. G. Mitchell (ㄷ) https://orcid.org/0000-0003-1960-2119

A. J. Coates 낭 https://orcid.org/0000-0002-6185-3125

Y. Wei (i) https://orcid.org/0000-0001-7183-0229

M. K. Dougherty (1) https://orcid.org/0000-0002-9658-8085

\section{References}

Achilleos, N., Guio, P., \& Arridge, C. S. 2010, MNRAS, 401, 2349 Arridge, C., Russell, C., Khurana, K., et al. 2008, JGRA, 113, A04214 Arridge, C. S., Jasinski, J. M., Achilleos, N., et al. 2016, JGRA, 121, 3006 Bader, A., Badman, S. V., Kinrade, J., et al. 2019a, JGRA, 124, 952
Bader, A., Badman, S. V., Yao, Z. H., Kinrade, J., \& Pryor, W. R. 2019b, JGRA, 124, 2451

Boström, R. 1964, JGR, 69, 4983

Carbary, J. F., Mitchell, D. G., Rymer, A. M., et al. 2017, in Geophysical Monograph Series, Dawn-Dusk Asymmetries in Planetary Plasma Environments, 230, ed. S. Haaland, A. Runov, \& C. Forsyth (Washington, DC: American Geophysical Union), 323

Delamere, P., Otto, A., Ma, X., Bagenal, F., \& Wilson, R. 2015, JGRA, 120, 4229 Dougherty, M. K., Cao, H., Khurana, K. K., et al. 2018, Sci, 362, 46

Dougherty, M. K., Kellock, S., Southwood, D. J., et al. 2004, SSRv, 114, 331 Esposito, L. W., Barth, C. A., Colwell, J. E., et al. 2004, SSRv, 115, 299

Forsyth, C., Fazakerley, A. N., Rae, I. J., et al. 2014, JGRA, 119, 927

Guo, R., Yao, Z., Wei, Y., et al. 2018a, NatAs, 2, 640

Guo, R. L., Yao, Z. H., Sergis, N., et al. 2018b, ApJL, 868, L23

Guo, R. L., Yao, Z. H., Sergis, N., et al. 2019, ApJL, 884, L14

Gurnett, D. A., Kurth, W. S., Kirchner, D. L., et al. 2004, SSRv, 114, 395

Gurnett, D. A., Shawhan, S. D., \& Shaw, R. R. 1983, JGRA, 88, 329

Krimigis, S. M., Mitchell, D. G., Hamilton, D. C., et al. 2004, SSRv, 114, 233

Liang, J., Donovan, E. F., Liu, W. W., et al. 2008, GeoRL, 35, L17S19

Liu, J., Angelopoulos, V., Chu, X., Zhou, X.-Z., \& Yue, C. 2015, GeoRL, 42,1669 
Lui, A. T. Y. 2004, SSRv, 113, 127

Masters, A., Achilleos, N., Kivelson, M. G., et al. 2010, JGRA, 115, A07225

McPherron, R. L., Russell, C. T., \& Aubry, M. P. 1973, JGR, 78, 3131

Mitchell, D., Krimigis, S., Paranicas, C., et al. 2009, P\&SS, 57, 1732

Mitchell, D. G., Carbary, J. F., Bunce, E. J., et al. 2016, Icar, 263, 94

Palmaerts, B., Radioti, A., Roussos, E., et al. 2016a, JGRA, 121, 11952

Palmaerts, B., Roussos, E., Krupp, N., et al. 2016b, Icar, 271, 1

Palmaerts, B., Vogt, M. F., Krupp, N., Grodent, D., \& Bonfond, B. 2017, in

Geophysical Monograph Series, Dawn-Dusk Asymmetries in Planetary

Plasma Environments, 230, ed. S. Haaland, A. Runov, \& C. Forsyth

(Washington, DC: American Geophysical Union), 307

Palmaerts, B., Yao, Z. H., Sergis, N., et al. 2020, GeoRL, 47, e88810
Radioti, A., Grodent, D., Gérard, J. C., et al. 2013, GeoRL, 40, 4498

Radioti, A., Grodent, D., Gérard, J. C., et al. 2015, JGRA, 120, 8633

Radioti, A., Yao, Z., Grodent, D., et al. 2019, ApJL, 885, L16

Rae, I. J., Mann, I. R., Angelopoulos, V., et al. 2009, JGRA, 114, A00C09

Rostoker, G. 1991, GMS, 64, 61

Rusaitis, L., Khurana, K. K., Kivelson, M. G., \& Walker, R. J. 2021, GeoRL, 48, e90967

Vasyliunas, V. 1983, Physics of the Jovian Magnetosphere (Cambridge: Cambridge Univ. Press)

Yao, Z., Radioti, A., Rae, I., et al. 2017, GeoRL, 44, 11217

Yao, Z. H., Radioti, A., Grodent, D., et al. 2018, JGRA, 123, 8502

Young, D. T., Berthelier, J. J., Blanc, M., et al. 2004, SSRv, 114, 1 